\title{
Longitudinal Analysis of the Absence of Intraoperative Neural Response Telemetry in Children using Cochlear Implants
}

\author{
Amanda Christina Gomes de Moura ${ }^{1}$ Maria Valéria Schmidt Goffi-Gomez ${ }^{1}$ Maria Ines Vieira Couto ${ }^{2}$ \\ Rubens Brito ${ }^{1}$ Robinson Koji Tsuji ${ }^{1}$ Debora Maria Befi-Lopes ${ }^{2}$ Carla Gentile Matas ${ }^{2}$ \\ Ricardo Ferreira Bento ${ }^{1}$
}

${ }^{1}$ ENT Department, Faculdade de Medicina da Universidade de São Paulo, São Paulo, São Paulo, Brazil

2 Physical Therapy, Speech, Occupational Therapy Department Faculdade de Medicina da Universidade de São Paulo, Universidade de São Paulo, São Paulo, Brazil
Address for correspondence Amanda Christina de Moura, AuD, Grupo de Implante Coclear, Hospital das Clínicas da Faculdade de Medicina da Universidade de São Paulo, Av. Dr. Enéas de Carvalho Aguiar, 255, São Paulo, São Paulo 05403-900, Brazil (e-mail: amandacgdm@gmail.com).

Int Arch Otorhinolaryngol 2014;18:362-368.

\begin{abstract}
Keywords

- cochlear implants

- neural response telemetry

- children

Introduction Currently the cochlear implant allows access to sounds in individuals with profound hearing loss. The objective methods used to verify the integrity of the cochlear device and the electrophysiologic response of users have noted these improvements.

Objective To establish whether the evoked compound action potential of the auditory nerve can appear after electrical stimulation when it is absent intraoperatively.

Methods The clinical records of children implanted with the Nucleus Freedom (Cochlear Ltd., Australia) (CI24RE) cochlear implant between January 2009 and January 2010 with at least 6 months of use were evaluated. The neural response telemetry (NRT) thresholds of electrodes $1,6,11,16$, and 22 during surgery and after at least 3 months of implant use were analyzed and correlated with etiology, length of auditory deprivation, and chronological age. These data were compared between a group of children exhibiting responses in all of the tested electrodes and a group of children who had at least one absent response.

Results The sample was composed of clinical records of 51 children. From these, 21\% (11) showed no NRT in at least one of the tested electrodes. After an average of 4.9 months of stimulation, the number of individuals exhibiting absent responses decreased from 21 to $11 \%(n=6)$.

Conclusion It is feasible that absent responses present after a period of electrical stimulation. In our sample, $45 \%(n=5)$ of the patients with intraoperative absence exhibited a positive response after an average of 4.9 months of continued electrical stimulation.
\end{abstract}

received

January 21, 2014

accepted

February 10, 2014

published online

July 17, 2014
DOI http://dx.doi.org/

10.1055/s-0034-1372510. ISSN 1809-9777.
Copyright $(\underset{0}{ } 2014$ by Thieme Publicações License terms

Ltda, Rio de Janeiro, Brazil
()(1) $\Theta \circledast$ 


\section{Introduction}

Severe or profound sensorineural hearing loss causes several deficiencies that are not only sensorial but also social and emotional. Characterized by a lack of perception and speech coding, individuals with this degree of hearing loss might need special resources to minimize the consequences of such a loss.

With the advancement of medicine and technology, the cochlear implant $(\mathrm{CI})$ represents an alternative that allows access to the sounds of speech in individuals with severe to profound hearing loss. ${ }^{1}$ Such improvements can also be observed in the objective methods used to verify the integrity of the cochlear device and the electrophysiologic response of users.

Neural response telemetry (NRT), developed from the studies by Abbas et $a{ }^{2}{ }^{2}$ is a fast and easily applicable technique that assesses the response of the peripheral segment of the auditory nerve to electrical stimulation. The implant used for NRT elicits stimuli and records the electrically evoked compound action potential (ECAP). Given that this is a fast and easily applicable technique, NRT is also used in the intraoperative setting. The major contribution of NRT is the confirmation of the physiologic integrity of the auditory nerve. $^{3}$ It is also useful when establishing the electrodes that might be included in a given map, the best stimulation speeds, the speech coding strategies, and the estimation of the stimulation of $\mathrm{T}$ (minimum stimulation) and $\mathrm{C}$ (maximum stimulation) levels. ${ }^{4}$

Composed of a negative peak (N1) followed by a positive peak (P2), the ECAP is analyzed with regard to the amplitude of the response. The measurement between $\mathrm{N} 1$ and $\mathrm{P} 2$ yields the wave amplitude, which varies according to the increased stimulation intensity. The ECAP represents the synchrony of a group of neurons, and the amplitude of response is proportional to the number of neurons activated by a stimulus. Consequently, the presence of the ECAP allows one to predict a satisfactory postoperative performance, ${ }^{5}$ which likely corresponds to better synaptic efficiency and synchronization of the neural response. ${ }^{6}$

Knowledge of the physiology and responses of the auditory nerve to electrical stimuli is important in establishing the current level used for stimulation and other programming adjustments. ${ }^{7}$ In some cases, the ECAP is not present during surgery, which might suggest a dysfunction of the cochlear nerve and existing neural structures. In some of these cases, continual stimulation predicts the appearance of neural response, which might be related to the synchronization of nerve fibers. ${ }^{8}$

Cafarelli Dees et $\mathrm{al}^{9}$ and van Dijk et al ${ }^{10}$ suggested the rates of postoperative presence of ECAP to be 96 and $90 \%$, respectively, whereas Guedes et al reported a rate of $80 \%{ }^{3}$ Guedes et al found that the absence of responses was correlated with limited prognosis. ${ }^{11}$ The continued use of CIs has tended to result in the emergence of the action potential after some months of stimulation. Even longitudinal studies on neural responses, however, have failed to mention the possibility of the appearance of a response or the average time required for the onset of such a response.

This study aimed at establishing whether the ECAP of the auditory nerve, when absent intraoperatively, can appear after continual electrical stimulation. When NRT responses were absent during surgery, we analyzed the evolution of these responses after at least 3 months of stimulation.

\section{Materials and Methods}

This study was approved by the Ethics Committee of Research Projects (CAPPesq) of the Clinical Board of the Clinical Hospital and the Medicine Faculty of the Universidade de São Paulo, protocol no. 010/11. This was a retrospective study conducted through the survey and analysis of the database of the Cochlear Implant Group of the Clinical Hospital of the Medicine Faculty of the Universidade de São Paulo (HC-FMUSP).

\section{Case Series}

We selected the clinical records of children who underwent multichannel CI (Nucleus Freedom, Cochlear Ltd., Australia, model CI24RE) surgery between January 2009 and January 2010 at the Cochlear Implant Group of the HC-FMUSP. Sixty-three children received CIs during that period. The criteria for sample selection included systematic use of the multichannel $\mathrm{CI}$ (more than $8 \mathrm{~h} / \mathrm{d}$ ), the use of the CI24RE internal and external units, and NRT records during the intraand postoperative periods for 6 months after surgery for electrodes $1,6,11,16$, and 22 . Of the 63 children, 51 met the inclusion criteria, with ages at implantation varying between 11 and 187 months.

\section{Procedures}

To record the NRT, the Cochlear Implant Group of the HC-FMUSP used a speech processor, an external magnet antenna, a link wire between the speech processor and the external antenna, a programming interface (Pod), and a computer to send and receive the neural data. All data were analyzed using the NRT software Custom Sound EP 2.0 (Cochlear Ltd., Australia), which controls the parameters of ECAP stimulation and recording.

The NRT results were analyzed according to the protocol described by Abbas et $\mathrm{al}^{2}$ and van Dijk et al. ${ }^{10}$ ECAP comprises a negative peak (N1) with an approximate latency between 0.2 and 0.4 milliseconds, followed by a positive peak (P2) with an approximate latency up to 1 millisecond. The amplitude of the response (measurement between N1 and P2) is proportional to the increase in the intensity of stimulation, which is measured in current units (CU). The presence of a visible N1 peak accompanied by the reproducibility of tracing, lack of artifact, or saturation of the amplifier (- Figs. 1 and $\mathbf{2}$ ) was considered a valid neural response. ${ }^{12}$ The values of positive responses represented the NRT thresholds expressed as CU, which is the smallest amount of current needed to generate an ECAP with an amplitude measurable by the software according to the autotelemetry protocol of van Dijk et al. ${ }^{10}$

Data regarding the etiology, model of electrodes, chronological age, and length of auditory deprivation were collected from the clinical records. It should be emphasized that the length of auditory deprivation is equal to the length of time between the onset of hearing loss and implant surgery. According to the intraoperative response, the children were separated into two groups. One group had positive neural 

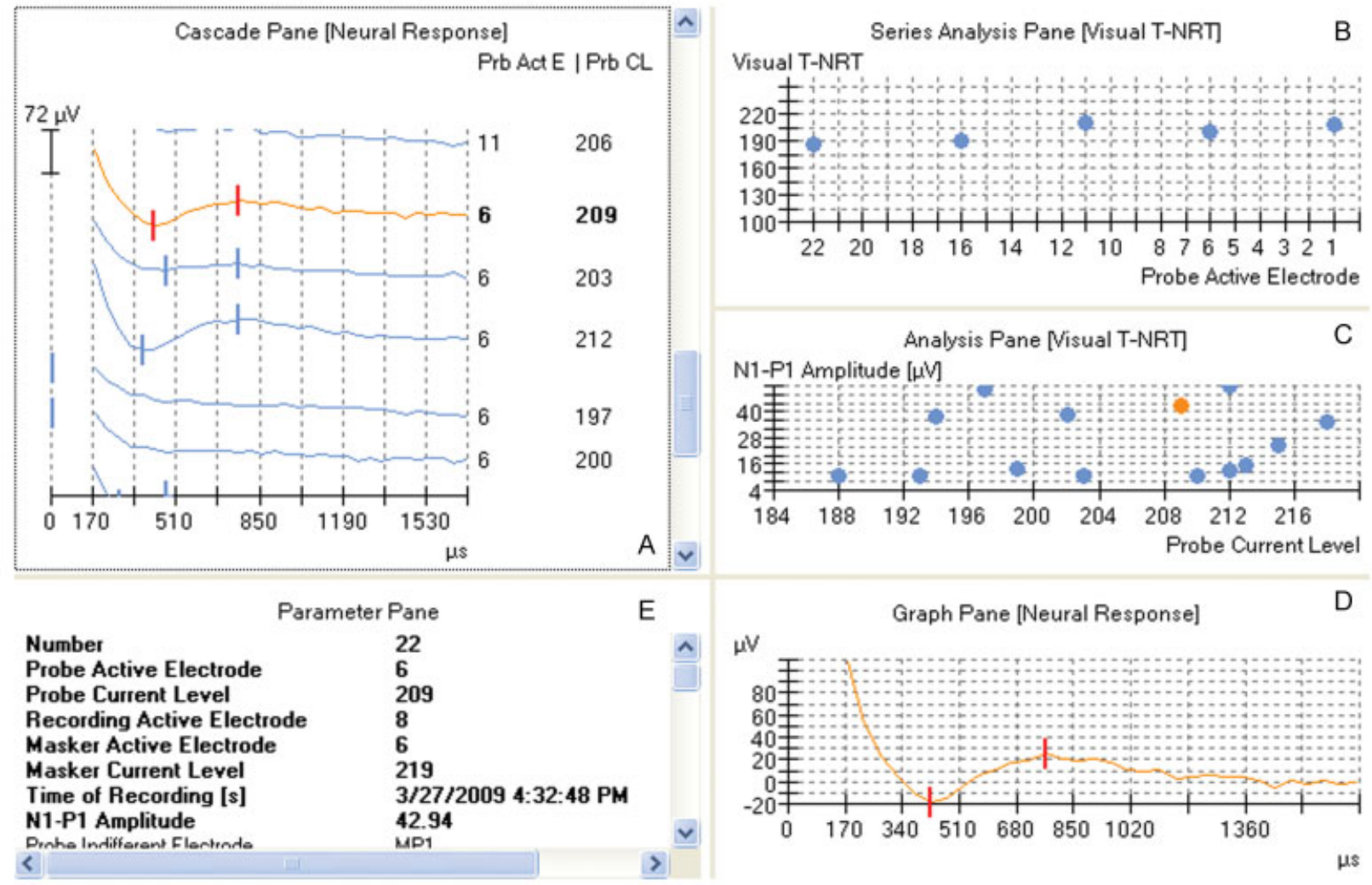

Fig. 1 Present response. (A) Stimulation and recording parameters employed for the measurement of the ECAP on electrode 6 and the stimulation level equal to $209 \mathrm{CU}$. (B) Different ECAP amplitudes obtained by various stimulation levels. (C) ECAP amplitudes as a function of the stimulation levels. (D) Regression curve supplied by the software. (E) ECAP response present at $209 \mathrm{CU}$ with $42.94 \mu \mathrm{V}$ of amplitude. Abbreviations: CMV, cytomegalovirus; CU, current units; ECAP, evoked compound action potential; NRT, neural response telemetry.

responses in all electrodes, and the other group exhibited an absence of response in at least one electrode. The NRT records were collected during surgery and at least 3 months after the activation of the $\mathrm{CI}$.

The average length of stimulation was estimated from the record of a postoperative time per individual. Patients returned for follow-up at $\sim 3$ and 6 months after activation, and the NRT response at the second or third times was used depending on the availability of records in the database.

\section{Statistical Analysis}

The values obtained for the threshold of neural responses per electrode $(1,6,11,16$, and 22$)$ were initially analyzed in a descriptive manner. The Mann-Whitney test was used to establish the relationships between the absence of response and age and the absence of response and length of deprivation. The Wilcoxon matched pairs test was used to analyze the appearance of postoperative NRT responses per electrode. Values of $p$ less than 0.05 were considered statistically significant.

\section{Results}

Of the 51 children, 40 exhibited positive responses during intraoperative NRT in all of the tested electrodes, whereas 11 children exhibited an absence of response in at least one electrode ( - Table 1). Regarding the implant model CI24RE, all patients received the perimodiolar electrode (Contour Advance), except for one child who received the CI24RE implant with a straight electrode beam. Regarding the causes of hearing loss found in the total sample (-Fig. $\mathbf{3}$ ), both groups exhibited a heterogeneous distribution in which an unknown etiology predominated.

Although $21 \%$ of the children showed an absence of response in at least one electrode, a positive intraoperative neural response was observed in all cases. The absence of an intraoperative NRT was observed in 16\% (16) of 255 electrodes tested (-Table $\mathbf{2}$ ). The absence was most frequent in the basal electrodes, primarily in electrode $1(18 \%)$ compared with electrodes 6, 16, and 22 (4\%) and electrode 11 (2\%).

After an average of 4.9 months of continual stimulation, the number of individuals in the total sample exhibiting an absence of response decreased from $21 \%$ (11) to $11 \%$ (6), thus reducing the relative absence/individual ratio by $18 \%$. Within the group exhibiting an intraoperative absence of response, the presence of NRT responses appeared in $45 \%$ (5) of the individuals and 14\% (8) of the tested electrodes. Among the positive responses, three appeared in patients with hearing loss due to unknown etiology, in one patient with hearing loss due to meningitis, and in one patient with hearing loss due to genetic causes. The results of the Mann-Whitney test indicated that age at implantation ( $U=184.500, z \leq 814, p<0.05$ and length of deprivation 

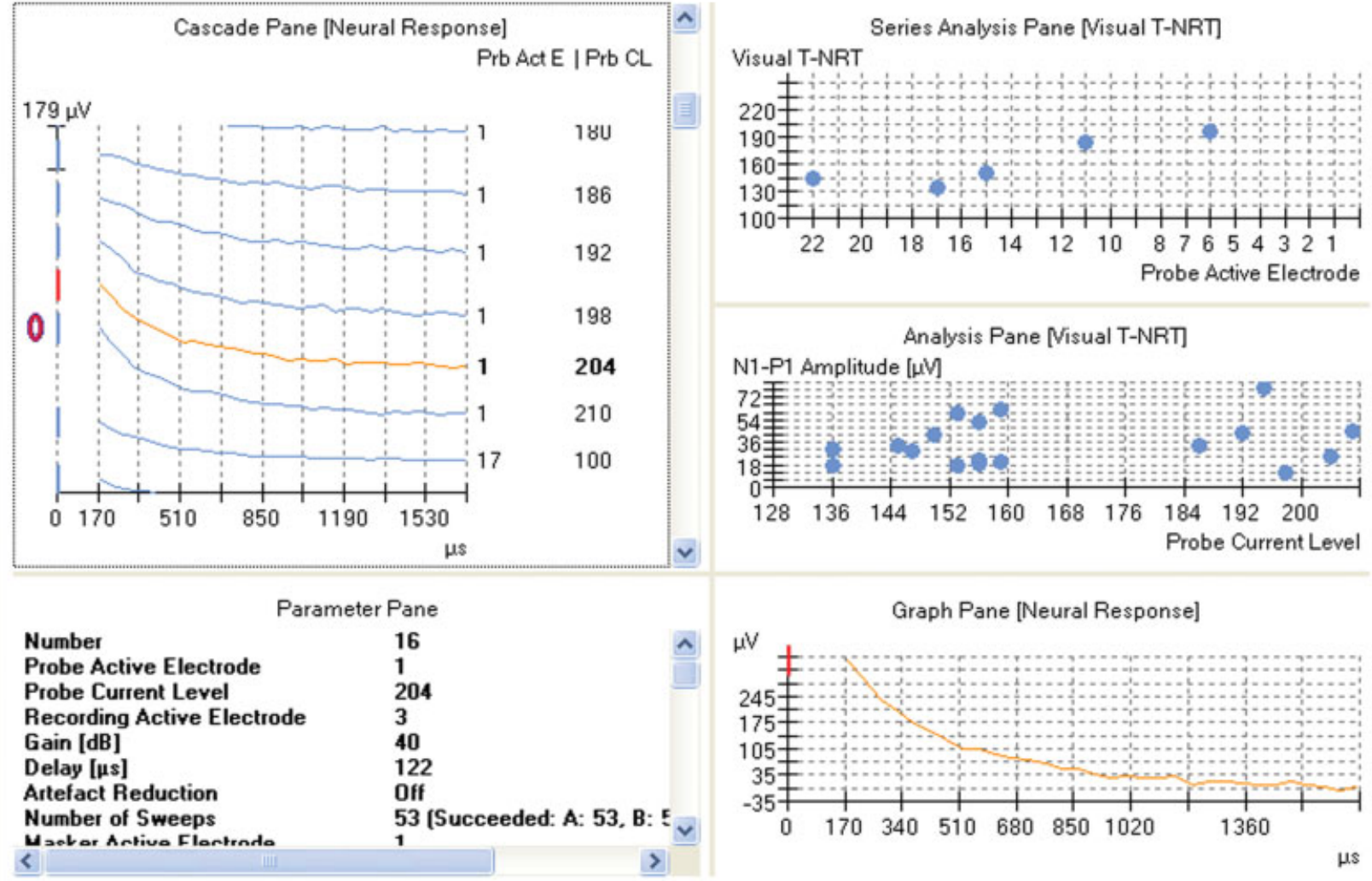

Fig. 2 Patient with hearing loss. Stimulation and recording parameters employed for the measurement of the evoked compound action potential (ECAP) on electrode 1 and stimulation level equal to 204 current units (CU). (B) Different ECAP amplitudes obtained by various stimulation levels. (C) ECAP amplitudes as a function of the stimulation levels. (D) Regression curve supplied by the software. (E) Absent ECAP response at the highest acceptable level within compliance.

$(U=177.000, z \leq 986, p<0.05$ were not statistically significant between the groups.

- Table 3 shows the comparison between the thresholds of the intra- and postoperative neural response in all of the tested electrodes in the group exhibiting an intraoperative absence of response and the time when the postoperative response was recorded. Electrode 1, which exhibited the highest rate of absence ( $82 \%$ ), was excluded from the analysis due to the low number of present responses. A Wilcoxon matched pairs test showed that the postoperative appearance of NRT responses was not statistically significant in any of the tested electrodes. There was, however, a tendency toward significance in electrode 22, which might be confirmed by studying a larger sample.

\section{Discussion}

Periodic NRT testing can be a helpful tool in clinical practice for determining the readaptation and recuperation of the cochlear nerve fibers after continued stimulation. In our study, $21 \%$ of the children exhibited an intraoperative absence of response to NRT. After an average of 4.9 months of continual stimulation, NRT responses appeared in $45 \%$ of individuals who had an absence of response during surgery.

Table 1 Sample distribution according to the presence (group P) or absence (group A) of response to intraoperative NRT according to age at implantation, length of deprivation, and model of electrode array

\begin{tabular}{|l|l|l|l|}
\hline & Group P & Group A & Total \\
\hline Age at Cl (mo) $( \pm$ SD) & $36.75( \pm 18.28)$ & $66.91( \pm 56.60)$ & $43.25( \pm 32.53)$ \\
\hline Time of deprivation $(\mathrm{mo})( \pm \mathrm{SD})$ & $34.85( \pm 18.18)$ & $64.82( \pm 56.17)$ & $41.31( \pm 32.30)$ \\
\hline Electrodes $(n)$ & 39 & 11 & 50 \\
\hline Perimodiolar & 1 & 0 & 1 \\
\hline Straight & 40 & 11 & 51 \\
\hline Total & \multicolumn{3}{|l|}{} \\
\hline
\end{tabular}

Abbreviations: $\mathrm{Cl}$, cochlear implantation; NRT, neural response telemetry; SD, standard deviation. 


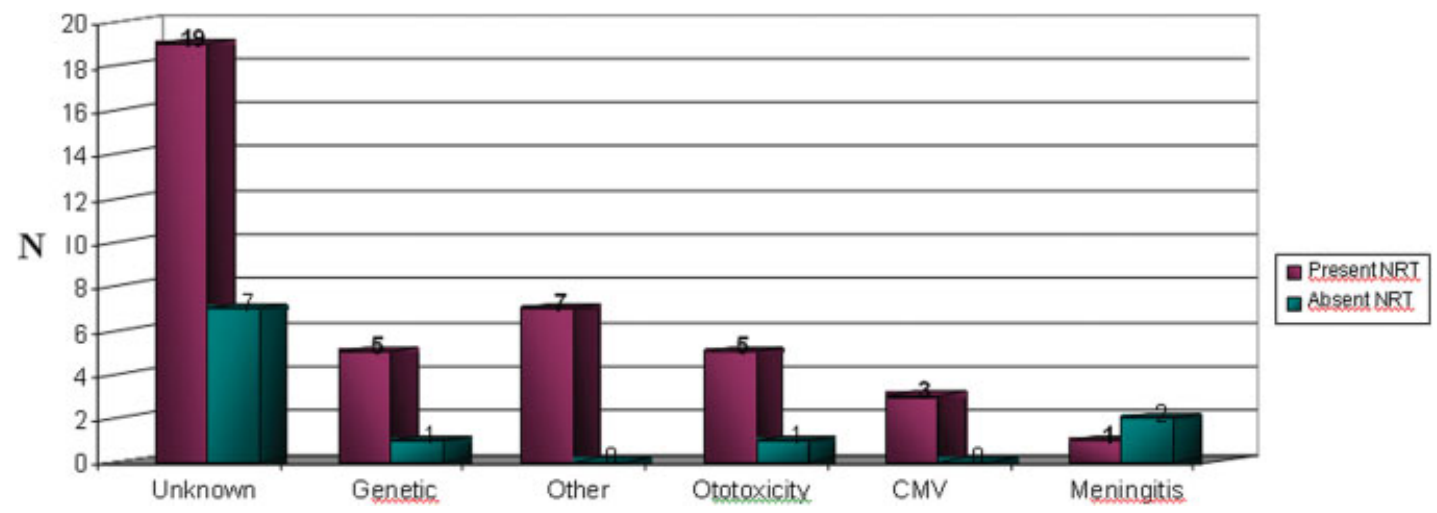

Fig. 3 Distribution of etiology in the total sample between the group with positive intraoperative neural response telemetry (NRT) response in all tested electrodes and the group with absent intraoperative NRT response in at least one tested electrode.

This finding suggests a tendency for the response to appear after continual use of the $\mathrm{CI}$, which might be related to the hypothesis that electrical stimulation causes alterations in the synaptic activity and supplies the auditory nerves with reinforced neurotrophic support. ${ }^{13,14}$ As a result of such modifications, it is believed that the synchronization of neural fibers occurs more efficiently due to the manner in which stimulation activates the primary neurons as well as causing a possible reduction in the periods of neuronal firing. ${ }^{15}$

Age at implantation and length of hearing loss, which are variables that often interfere with the determination of resources and the programming of the $\mathrm{Cl}$ in clinical practice, did not exhibit any significant influence on the absence of

Table 2 Distribution of the presence and absence of intraoperative response in 255 tested electrodes

\begin{tabular}{|l|l|l|l|l|l|l|}
\hline & $\begin{array}{l}\text { Electrode 1, } \\
\boldsymbol{n}(\%)\end{array}$ & $\begin{array}{l}\text { Electrode 6, } \\
\boldsymbol{n}(\%)\end{array}$ & $\begin{array}{l}\text { Electrode 11, } \\
\boldsymbol{n}(\%)\end{array}$ & $\begin{array}{l}\text { Electrode 16, } \\
\boldsymbol{n}(\%)\end{array}$ & $\begin{array}{l}\text { Electrode 22, } \\
\boldsymbol{n}(\%)\end{array}$ & $\begin{array}{l}\text { Total, } \\
\boldsymbol{n}(\%)\end{array}$ \\
\hline Present & $42(82)$ & $49(96)$ & $50(98)$ & $49(96)$ & $49(96)$ & $239(94)$ \\
\hline Absent & $9(18)$ & $2(4)$ & $1(2)$ & $2(4)$ & $2(4)$ & $16(6)$ \\
\hline Total & $51(100)$ & $51(100)$ & $51(100)$ & $51(100)$ & $51(100)$ & $255(100)$ \\
\hline
\end{tabular}

Table 3 NRT thresholds in an intra- and postoperative setting in individuals exhibiting at least one absence of response during surgery

\begin{tabular}{|c|c|c|c|c|c|c|c|c|c|c|c|}
\hline ID & \multicolumn{2}{|c|}{ Electrode 1} & \multicolumn{2}{|c|}{ Electrode 6} & \multicolumn{2}{|c|}{ Electrode 11} & \multicolumn{2}{|c|}{ Electrode 16} & \multicolumn{2}{|c|}{ Electrode 22} & \multirow[b]{2}{*}{$\begin{array}{l}\text { Time } \\
\text { postop (mo) }\end{array}$} \\
\hline & Intraop & Postop & Intraop & Postop & Intraop & Postop & Intraop & Postop & Intraop & Postop & \\
\hline 6 & 198 & 128 & 213 & 146 & 234 & 166 & 245 & 167 & $\downarrow$ & 146 & 6 \\
\hline 7 & $\downarrow$ & 190 & $\downarrow$ & 152 & 196 & 157 & $\downarrow$ & 170 & 166 & 130 & 4 \\
\hline 19 & $\downarrow$ & $\downarrow$ & 202 & 187 & 204 & 184 & 203 & 184 & 197 & 166 & 6 \\
\hline 22 & $\downarrow$ & 214 & 195 & 163 & 218 & $\downarrow$ & 222 & $\downarrow$ & $\downarrow$ & 145 & 5 \\
\hline 28 & $\downarrow$ & 211 & 181 & 187 & 157 & 154 & 163 & 166 & 193 & 166 & 6 \\
\hline 29 & $\downarrow$ & $\downarrow$ & 178 & 178 & 183 & 202 & 179 & 205 & 182 & 190 & 6 \\
\hline 30 & $\downarrow$ & $\downarrow$ & 199 & 207 & 178 & 192 & 186 & 132 & 200 & 159 & 4 \\
\hline 35 & $\downarrow$ & $\downarrow$ & 214 & 209 & 189 & 184 & 206 & 194 & 185 & 178 & 3 \\
\hline 36 & $\downarrow$ & $\downarrow$ & $\downarrow$ & $\bar{\downarrow}$ & $\downarrow$ & $\downarrow$ & 194 & 211 & 197 & 188 & 4 \\
\hline 37 & 178 & 187 & 187 & 202 & 187 & 202 & $\downarrow$ & 202 & 148 & 175 & 4 \\
\hline 44 & $\downarrow$ & $\downarrow$ & 211 & 193 & 204 & 199 & 202 & 190 & 176 & 175 & 6 \\
\hline
\end{tabular}

Abbreviations: Intraop, intraoperatively; Postop, postoperatively; NRT, neural response telemetry; SD, standard deviation.

Note: All responses are given in current units; $\downarrow$ indicates the absence of a response. Analyzing whether the presence (or appearance) of a response is significant in any electrode. 
NRTs or in the later appearance of response. In our study, these results can be explained by the fact that the sample was comprised exclusively of children between the ages of 11 and 187 months. Similar studies should be performed with adults to analyze the possible relationship between age at implantation, length of hearing loss, and absence of intra- and postoperative ECAP responses.

In our case series, hearing loss due to unknown causes was the prevailing etiology. Due to the small number of individuals lacking an NRT response, the results reported in prior studies, ${ }^{3,10}$ which indicate a prevalence in the absence of response in all electrodes in progressive and ossifying conditions (meningitis and otosclerosis), could not be confirmed. This is likely because in the present study, only children were analyzed, and otosclerosis is known to be predominantly present in the adult population.

Electrodes 1, 6, 11, 16, and 22 were tested using the default settings in the Custom Sound (Cochlear) software, which is programmed to record the NRT responses from the bundle of electrodes allocated in the cochlea. The recorded responses therefore must have originated from every cochlear area to detect possible tonotopic interferences in the ECAP responses. Only individuals with an impedance telemetry within the normal range (above $0.7 \mathrm{k} \Omega$ and below $20 \mathrm{k} \Omega$ ) were analyzed, thus avoiding the integrity of the chain of electrodes from becoming a defining variable in the recording of the NRT responses.

We observed a tendency for the response to be absent at higher current levels in the basal electrodes, which is consistent with previous studies. ${ }^{3,16-18}$ This absence is possibly associated with the position of the electrodes on the basal turn, which becomes more distant from the modiolus (due to the position of cochleostomy) and leads to the need for higher levels of energy in this area. ${ }^{19}$ In this case, the position of cochleostomy would distance the array of electrodes from the modiolus. It would, however, be interesting to study the influence of electrode position on the threshold of neural responses in implanted patients using the technique of insertion through a cochleostomy and a round window with straight and perimodiolar electrodes. In our case series, only one child had a straight electrode implant, and this child exhibited responses in all of the tested electrodes. Other studies have suggested a relationship between residual hearing and the need of lower levels of current required to elicit a response in these areas, possibly explaining the need for higher levels of energy in the basal electrodes (an area with less auditory residue). ${ }^{2,20}$

- Table 3 shows that in individual 22, the postoperative record analysis showed the appearance of NRT responses in electrodes that were absent from the intraoperative setting (1 and 22). It was, however, possible to observe that two of the electrodes (11 and 16) began to exhibit absent responses. There are no data in the literature explaining this phenomenon. Further studies are therefore needed to analyze the frequency and causes of these events.

The results of our study highlight the importance of conducting intraoperative NRT examinations, which is a fast and simple procedure that can ensure the physiological integrity of the cochlear nerve and allow for the monitoring of the response over time. In addition, this method can assist in the programming of the speech processor of the CI. Periodic retesting of ECAP responses allows for a deeper understanding of the neural physiology of patients presenting with an absence of response and may assist in the development of better coding strategies for the device and more efficient rehabilitation for each individual.

\section{Conclusion}

ECAPs of the auditory nerve appeared in $45 \%$ of individuals with intraoperative absence of response after an average of 4.9 months of continual stimulation.

\section{References}

1 Ferrari DV, Sameshima K, Costa Filho OA, Bevilacqua MC. A telemetria de respostas neurais no sistema de implante coclear multicanal nucleus 24: revisão da literatura. Rev Bras Otorrinolaringol 2004;70(1):112-118

2 Abbas PJ, Brown CJ, Shallop JK, et al. Summary of results using the nucleus CI24M implant to record the electrically evoked compound action potential. Ear Hear 1999;20(1):45-59

3 Guedes MC, Brito Neto RV, Goffi-Gomez MVS, et al. Telemetria de resposta neural intra-operatória em usuários de implante coclear. Rev Bras Otorrinolaringol 2005;71(5):660-667

4 Shallop JK, Facer GW, Peterson A. Neural response telemetry with the nucleus CI24M cochlear implant. Laryngoscope 1999;109(11): $1755-1759$

5 Gantz BJ, Brown CJ, Abbas PJ. Intraoperative measures of electrically evoked auditory nerve compound action potential. Am J Otol 1994;15(2):137-144

6 Blamey P. Are spiral ganglion cell numbers important for speech perception with a cochlear implant? Am J Otol 1997;18(6, Suppl): S11-S12

7 Abbas PJ, Brown CJ. Electrophysiology and device telemetry. In: Waltzman SB, Cohen NL, eds. Cochlear Implants. New York, NY: Thieme; 2000:121-138

8 Tanamati LF, Bevilacqua MC, Costa OA. Avaliação longitudinal do ECAP registrado em crianças usuárias de implante coclear. Rev Bras Otorrinolaringol 2009;75(1):90-98

9 Cafarelli Dees D, Dillier N, Lai WK, et al. Normative findings of electrically evoked compound action potential measurements using the neural response telemetry of the Nucleus CI24M cochlear implant system. Audiol Neurootol 2005;10(2):105-116

10 van Dijk B, Botros AM, Battmer RD, et al. Clinical results of AutoNRT, a completely automatic ECAP recording system for cochlear implants. Ear Hear 2007;28(4):558-570

11 Guedes MC, Weber R, Goffi-Gomez MVS, Brito Neto RV, Peralta CGO, Bento RF. Efeitos do potencial de ação neural sobre a percepção de fala em usuários de implante coclear. Rev Bras Otorrinolaringol 2007;73(4):439-445

12 Brown CJ. Clinical uses of electrically evoked auditory nerve and brainstem responses. Curr Opin Otolaryngol Head Neck Surg 2003; 11(5):383-387

13 Roehm PC, Hansen MR. Strategies to preserve or regenerate spiral ganglion neurons. Curr Opin Otolaryngol Head Neck Surg 2005; 13(5):294-300

14 Shepherd RK, Hardie NA. Deafness-induced changes in the auditory pathway: implications for cochlear implants. Audiol Neurootol 2001;6(6):305-318

15 Gordon KA, Papsin BC, Harrison RV. Activity-dependent developmental plasticity of the auditory brain stem in children who use cochlear implants. Ear Hear 2003;24(6):485-500 
16 Gordon KA, Ebinger KA, Gilden JE, Shapiro WH. Neural response telemetry in 12- to 24-month-old children. Ann Otol Rhinol Laryngol Suppl 2002;189(189):42-48

17 Hughes ML, Brown CJ, Abbas PJ, Wolaver AA, Gervais JP. Comparison of EAP thresholds with MAP levels in the nucleus 24 cochlear implant: data from children. Ear Hear 2000;21(2): 164-174

18 Smoorenburg GF, Willeboer C, van Dijk JE. Speech perception in nucleus $\mathrm{CI} 24 \mathrm{M}$ cochlear implant users with processor settings based on electrically evoked compound action potential thresholds. Audiol Neurootol 2002;7(6):335-347

19 Tsuji RK, Goffi-Gomez MVS, Peralta CO, et al. Neural response thresholds in the Nucleus Contour cochlear implant before and after stylet removal. Acta Otolaryngol 2009;129(11):1330-1336

20 Franck KH, Norton SJ. Estimation of psychophysical levels using the electrically evoked compound action potential measured with the neural response telemetry capabilities of Cochlear Corporation's CI24M device. Ear Hear 2001;22(4):289-299 